\title{
Entangling a series of trapped ions by moving cavity bus
}

\author{
ZHANG Miao, ${ }^{1}$ JIA Huan-Yu, ${ }^{1}$ and WEI Lian-Fu ${ }^{* 1,2}$ \\ ${ }^{1}$ Quantum Optoelectronics Laboratory, School of Physics and Technology, \\ Southwest Jiaotong University, Chengdu 610031, China \\ ${ }^{2}$ State Key Laboratory of Optoelectronic Materials and Technologies, \\ School of Physics and Engineering, Sun Yat-sen University, Guangzhou 510275, China
}

(Dated: December 25, 2018)

\begin{abstract}
Entangling multiple qubits is one of the central tasks for quantum information processings. Here, we propose an approach to entangle a number of cold ions (individually trapped in a string of microtraps) by a moved cavity. The cavity is pushed to include the ions one by one with an uniform velocity, and thus the information stored in former ions could be transferred to the latter ones by such a moving cavity bus. Since the positions of the trapped ions are precisely located, the strengths and durations of the ion-cavity interactions can be exactly controlled. As a consequence, by properly setting the relevant parameters typical multi-ion entangled states, e.g., $W$ state for 10 ions, could be deterministically generated. The feasibility of the proposal is also discussed.
\end{abstract}

PACS numbers: 42.50.Dv, 03.67.Bg, 37.30.+i

\footnotetext{
*Email:weilianf@mail.sysu.edu.cn; weilianfu@gmail.com
} 
Entanglement is one of the heart concepts in quantum mechanics, and has potential applications in high precision spectroscopy, quantum communication, cryptography and computation [1]. Entanglements of two and three particles have been studied extensively and are well understood. Recently, entanglements of more than three particles, e.g., four, five, six, seven, and eight ones, have been successively demonstrated [1-4]. Among the various kinds of entangled states, the wellknown $W$ state plays an important role in quantum information processes [5], as its entanglement is maximally persistent and robust even under particle losses [6]. Therefore, much interests have been paid recently on the generations of $W$ states (see, e.g. [4]).

To generate an entangled state, the system of trapped ions has many advantages, such as the convenient manipulations, relative long coherence time, and easily readout, etc. [7]. Actually, most of the above multi-particle entanglements (e.g., the eight ions) were first realized experimentally in such a system. Note that all these experiments were based on either Cirac-Zoller (CZ) [8] or Mølmer-Sørensen (MS) [9] models. There, a string of ions are trapped in a single ion-trap, the atomic levels of the trapped ions act as the qubits and the collective motions of the ions serve as the data bus. Assisted by such a data bus the trapped ions could be effectively entangled under the drivings of the applied laser beams [10].

Here, we propose an alternative approach to entangle a series of trapped ions by moving cavity bus, instead of the collective motions of the ions utilized in the famous CZ and MS models. The system we proposed here is schematized in Fig. 1, wherein cold ions are individually confined in a string of microtraps [11] and the inter-ion interactions are negligible. A high finesse cavity is moved to include the ions on the string one by one, and the cavity mode acts a data bus [12, 13] to transfer the information from one ion to the others. Comparing to the usual schemes (e.g., the $\mathrm{CZ}$ and MS models) using the moving cavity bus to generate multi-particle entanglement is conceptually simpler and obviously scalable.

We assume that the velocity of the cavity motion could be properly set, and thus the cavity could resonantly couple the ions one by one with the controllable durations. Also, the ions are assumed to be strongly trapped and well cooled, such that the regions of the ions' spatial wave packets are much smaller than the waist and wavelength of the resonant cavity [14-16]. Therefore, the strengths of the cavity-ion couplings are well-defined (i.e., decided by the located positions of the ions relative to the cavity mode [17]). We shall show that, by properly setting these experimental parameters, the multi-ion (e.g., from 2- to 10-ion) $W$ states could be prepared within the coherence times of the system. 


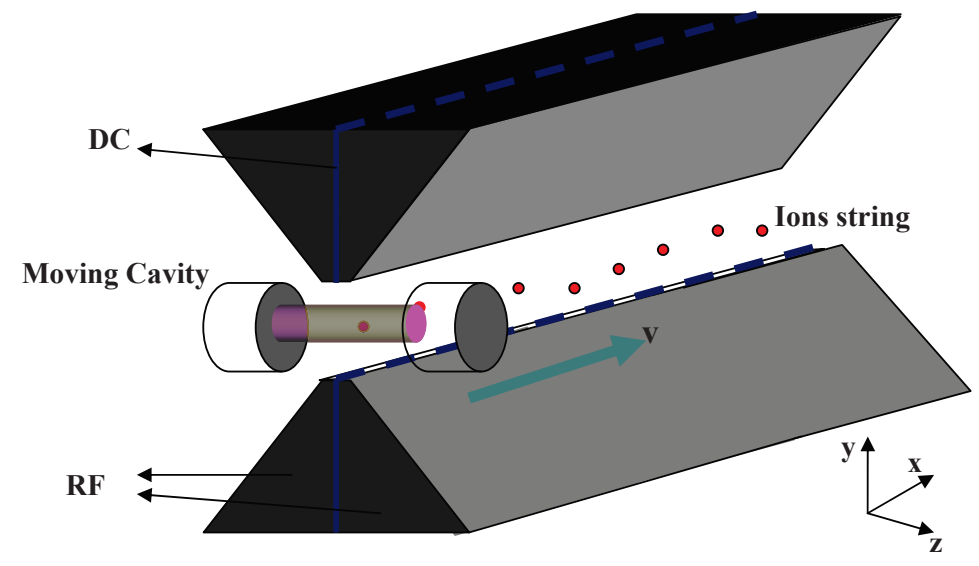

FIG. 1: (Color online) A sketch of the moving cavity bus to entangle a series of trapped ions, which are independently trapped in a string of microtraps.

Without loss of generality, we assume that the interaction between the moving cavity and the two-level ion is resonant and can be well described by the well-known Jaynes-Cummings model [12]

$$
\hat{H}_{\mathrm{JCM}}=\hbar \Omega(x, y, z)\left(\hat{a}^{\dagger} \hat{\sigma}_{-}+\hat{a} \hat{\sigma}_{+}\right) .
$$

Here, $\hat{a}^{\dagger}$ and $\hat{a}$ are the bosonic creation and annihilation operators of the cavity field, respectively. $\hat{\sigma}_{+}=|e\rangle\langle g|$ and $\hat{\sigma}_{+}=|e\rangle\langle g|$ are the raising and lowering operators of the trapped ion, respectively. The strength of the ion-field coupling $\Omega(x, y, z)=\Omega_{0} f(x, y, z)$ depends on the relative position $(x, y, z)$ of the trapped ion in the moving cavity, and $\Omega_{0}$ is a constant. Also, the cavity mode is the usual $\mathrm{TEM}_{00}$ mode with the distribution form [17]

$$
f(x, y, z)=e^{-\left(x^{2}+y^{2}\right) / w^{2}} \cos (2 \pi z / \lambda),
$$

where $w$ and $\lambda$ are the waist and wavelength of the cavity mode, respectively.

Initially, the system is prepared in the state $\left|\varphi_{0}\right\rangle=\left|g_{1} g_{2} g_{3} \cdots g_{n}\right\rangle|1\rangle$, i.e., all the trapped ions (where the index $n$ represents $n$th ion) are in their atomic ground states and the cavity is in its single-photon excited state. Now, we push the cavity (with an uniform velocity $v$ along the $x$ axis) to resonantly excite the trapped ions one by one. In this process, each ion interacts with the cavity field with an effective duration $t_{\text {eff }}=\sqrt{\pi} w / v$, and the cavity-ion coupling strength reads $\Omega=\Omega_{0} f(0, y, z)$. This is similar to that of the flying Rydberg atoms interacting with the cavity [12]. Therefore, in this process the system undergoes the following a series of evolutions:

Firstly, after the cavity excites the first trapped ion (with the cavity-ion interacting strength 
$\left.\Omega_{1}=\Omega_{0} f\left(0, y_{1}, z_{1}\right)\right)$, the system is evolved to the state

$$
\left|\varphi_{1}\right\rangle=\cos \left(\Omega_{1} t_{\mathrm{eff}}\right)\left|g_{1} g_{2} g_{3} \cdots g_{n}\right\rangle|1\rangle-i \sin \left(\Omega_{1} t_{\mathrm{eff}}\right)\left|e_{1} g_{2} g_{3} \cdots g_{n}\right\rangle|0\rangle .
$$

Secondly, after the cavity excites the second trapped ion (with the coupling strength $\Omega_{2}=$ $\left.\Omega_{0} f\left(0, y_{2}, z_{2}\right)\right)$, the state of system is

$$
\begin{aligned}
\left|\varphi_{2}\right\rangle= & \cos \left(\Omega_{1} t_{\mathrm{eff}}\right) \cos \left(\Omega_{2} t_{\mathrm{eff}}\right)\left|g_{1} g_{2} g_{3} \cdots g_{n}\right\rangle|1\rangle \\
& -i \cos \left(\Omega_{1} t_{\mathrm{eff}}\right) \sin \left(\Omega_{2} t_{\mathrm{eff}}\right)\left|g_{1} e_{2} g_{3} \cdots g_{n}\right\rangle|0\rangle-i \sin \left(\Omega_{1} t_{\mathrm{eff}}\right)\left|e_{1} g_{2} g_{3} \cdots g_{n}\right\rangle|0\rangle .
\end{aligned}
$$

Successively, after the $n$th trapped ion is excited (with the strength $\Omega_{n}=\Omega_{0} f\left(0, y_{n}, z_{n}\right)$ ) by the moving cavity, we have a $n$-ion entangled state

$$
\begin{aligned}
\left|\varphi_{n}\right\rangle= & -i\left(A_{1}\left|e_{1} g_{2} g_{3} \cdots g_{n}\right\rangle+A_{2}\left|g_{1} e_{2} g_{3} \cdots g_{n}\right\rangle+A_{3}\left|g_{1} g_{2} e_{3} \cdots g_{n}\right\rangle\right. \\
& \left.+\cdots \cdots+A_{n}\left|g_{1} g_{2} g_{3} \cdots e_{n}\right\rangle\right)|0\rangle+B\left|g_{1} g_{2} g_{3} \cdots g_{n}\right\rangle|1\rangle
\end{aligned}
$$

with the amplitudes

$$
A_{n}= \begin{cases}\sin \left(\Omega_{1} t_{\mathrm{eff}}\right) & \text { for } n=1, \\ \sin \left(\Omega_{n} t_{\mathrm{eff}}\right) \prod_{j=1}^{n-1} \cos \left(\Omega_{j} t_{\mathrm{eff}}\right) & \text { for } n \geq 2,\end{cases}
$$

and $B=\prod_{j=1}^{n} \cos \left(\Omega_{j} t_{\mathrm{eff}}\right)$. Obviously, for preparing the $N$-ion $W$ state, the above coefficients should be set as $A_{n}=1 / \sqrt{N}$ and $B=0$. This requires that the coupling strength $\Omega_{n}$ of $n$th ion interacting with the cavity should satisfy the condition:

$$
\Omega_{n}=\Omega_{0} f\left(0, y_{n}, z_{n}\right)=\frac{v}{w \sqrt{\pi}} \begin{cases}\arcsin \left(\frac{1}{\sqrt{n}}\right) & \text { for } n=1, \\ \arcsin \left[\tan \left(\Omega_{n-1} \sqrt{\pi} w / v\right)\right] & \text { for } n \geq 2 .\end{cases}
$$

This equation can be easily solved by numerical method. Table 1 shows some numerical results for generating the desirable $W$ states with $\Omega_{0} \sim 2 \pi \times 14.8 \mathrm{MHz}, w \sim 10 \mu \mathrm{m} \mathrm{[18,} \mathrm{19],} v=800 \mathrm{~m} / \mathrm{s}$ and $z=0$. Above, the ions are assumed to be confined in different position $\left(y_{n}, z=0\right)$ for achieving different ion-cavity coupling strength $\Omega_{n}$. Therefore, the ions should be precisely trapped in any proper positions, such that $\Omega_{n}$ are exactly controllable. Also, the velocity of the cavity should be set sufficiently precise to achieve the $W$ states with high fidelities.

Due to the practically-existing position-uncertain $(\delta y, \delta z)$ of the ion in the cavity and the velocity-fluctuation $\delta v$ of the cavity motion, the amplitudes $A_{n}$ of the generated $W$ states should not be exactly equal to the expected $1 / \sqrt{N}$. Instead, they might read

$$
A_{n}^{\text {flu }}= \begin{cases}\sin \left(\alpha_{1}\right) & \text { for } \quad n=1, \\ \sin \left(\alpha_{n}\right) \prod_{j=1}^{n-1} \cos \left(\alpha_{j}\right) & \text { for } \quad n \geq 2,\end{cases}
$$


TABLE I: Numerical solutions of $y_{n}(\mu \mathrm{m})$ for generating multi-ion $W$ states (from 2 to 10 ions) with $\Omega_{0}=2 \pi \times 14.8 \mathrm{MHz}, w=10 \mu \mathrm{m}, v=800 \mathrm{~m} / \mathrm{s}$, and $z=0$. Note that $y_{n}$ denotes the relative position of the $n$th ion in the y-axis.

\begin{tabular}{ccccccccccc}
\hline$N$ & $y_{1}$ & $y_{2}$ & $y_{3}$ & $y_{4}$ & $y_{5}$ & $y_{6}$ & $y_{7}$ & $y_{8}$ & $y_{9}$ & $y_{10}$ \\
\hline 2 & 9.8204 & 5.2083 & & & & & & & & \\
3 & 10.9918 & 9.8204 & 5.2083 & & & & & & \\
4 & 11.7042 & 10.9918 & 9.8204 & 5.2083 & & & & & & \\
5 & 12.2126 & 11.7042 & 10.9918 & 9.8204 & 5.2083 & & & & & \\
6 & 12.6058 & 12.2126 & 11.7042 & 10.9918 & 9.8204 & 5.2083 & & & & \\
7 & 12.9253 & 12.6058 & 12.2126 & 11.7042 & 10.9918 & 9.8204 & 5.2083 & & & \\
8 & 13.1936 & 12.9253 & 12.6058 & 12.2126 & 11.7042 & 10.9918 & 9.8204 & 5.2083 & & \\
9 & 13.4244 & 13.1936 & 12.9253 & 12.6058 & 12.2126 & 11.7042 & 10.9918 & 9.8204 & 5.2083 & \\
10 & 13.6265 & 13.4244 & 13.1936 & 12.9253 & 12.6058 & 12.2126 & 11.7042 & 10.9918 & 9.8204 & 5.2083 \\
\hline
\end{tabular}

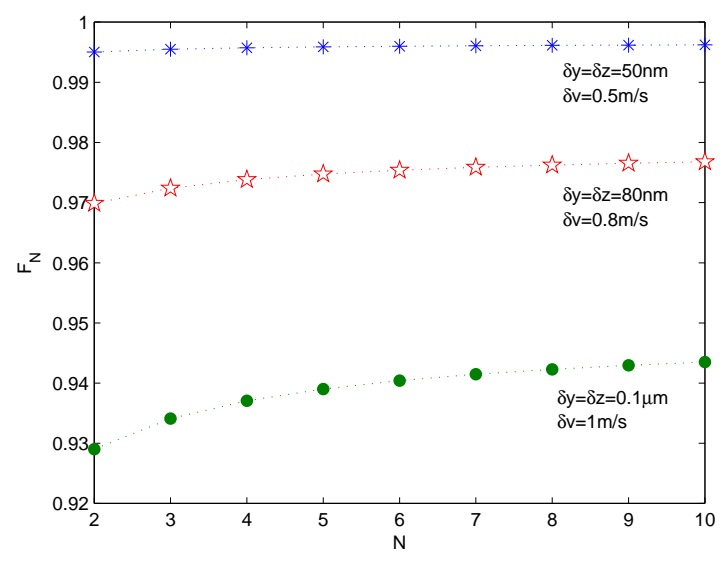

FIG. 2: The fidelities of the generated multi-ion $W$ states (from 2 to 10 ions).

with

$$
\alpha_{n}=\frac{\Omega_{0} \sqrt{\pi} w}{v \pm \delta v} e^{-\left(y_{n} \pm \delta y\right)^{2} / w^{2}} \cos \left[2 \pi\left(z_{n} \pm \delta z\right) / \lambda\right] .
$$

Therefore, the experimentally generated $W_{N}^{\text {flu }}$ states might not be the expected $W_{N}$ states. In order to describe these departures, a function $F_{N}=\left\langle W_{N} \mid W_{N}^{\text {flu }}\right\rangle=\sum_{n=1}^{N} A_{n} A_{n}^{\text {flu }}$ could be introduced to describe the fidelities of the generated $W$ states. Certainly, when $\delta v, \delta y, \delta z \rightarrow 0, A_{n}^{\text {flu }} \rightarrow 1 / \sqrt{N}$ 
and thus $F_{N} \rightarrow 1$.

In fact, confining the ions in an exceedingly small region, e.g., on the order of $10 \mathrm{~nm}$, has been demonstrated by a number of experiments (see, e.g., [14-16]). Following the typical experiment reported in Ref. [14], we consider that the cavity is resonant to excite the transition ${ }^{40} \mathrm{Ca}^{+}: \mathrm{P}_{1 / 2} \leftrightarrow$ $\mathrm{D}_{3 / 2}$, corresponding to a wavelength of $\lambda=866 \mathrm{~nm}$. Obviously, the spatial uncertain (e.g., $50 \mathrm{~nm}$ ) of the trapped ion is far less than this wavelength and the waist (with the order of $10 \mu \mathrm{m}$ ), and thus the uncertain of the coupling strength $\Omega_{n}$ is significantly small. Consequently, the fidelities of the generated $W$ states could be high. Fig. 2 shows some numerical results of fidelities $F_{N}$ with the typical parameters $\Omega_{0}=2 \pi \times 14.8 \mathrm{MHz}, w=10 \mu \mathrm{m}, v=800 \mathrm{~m} / \mathrm{s}$, and $z=0$. Certainly, for the relative smaller fluctuations $\delta v=0.5 \mathrm{~m} / \mathrm{s}$ and $\delta y=\delta z=50 \mathrm{~nm}$, the fidelities for the generated multi-ion $W$ states (from 2 to 10 ions) can reach to $99 \%$, as shown in Fig. 2.

In addition, the sizes of the experimental microtraps should be sufficiently small and the cavity should be pushed sufficiently quick, such that more trapped ions could be entangled within the finite coherence times of the trapped ions and the cavity. This is because the total time of the $N$ ions crossing the cavity depends on the distance $d$ (between the two neighboring trapped ions) and the velocity $v$ of the moving cavity, as $T=N d / v$. For example, the total time to generate the 10 -ion $W$ state can be less than $0.25 \mu$ s for $d=20 \mu \mathrm{m}$ and $v=800 \mathrm{~m} / \mathrm{s}$. Also, it has been experimentally demonstrated [11] that the sizes of the microtraps can be on the order of $10 \mu \mathrm{m}$ by the photolithography and metal deposition techniques. Thus, independently trapping the ions with sufficiently small distances, e.g., $20 \mu \mathrm{m}$, is feasible. By improving the experimental setup to obtain the moving cavity with any modest velocities (e.g., $800 \mathrm{~m} / \mathrm{s}$ ) and velocity-fluctuations (e.g., $0.5 \mathrm{~m} / \mathrm{s}$ ) are not exceedingly difficult, in principle. It has been shown that the spontaneous decay rate on the $\mathrm{P}_{1 / 2} \leftrightarrow \mathrm{D}_{3 / 2}$ is about $\Gamma=2 \pi \times 1.69 \mathrm{MHz}$ [14], and the decay rate of the cavity (with the finesse $\mathcal{F}=3.5 \times 10^{4}$ ) is about $\kappa=2 \pi \times 102 \mathrm{KHz}$ [16]. Not that the above time for entangling the ions could be further shorted by enhancing the coupling strength $\Omega_{0}$ and moving the cavity more quick. This implies that the present method to generate multi-ion entanglement within a finite coherence time is possibly feasible.

In conclusion, we have proposed an idea to entangle a series of trapped ions: pushing the cavity bus to resonantly excite the trapped ions one by one. We have shown that by confining the ions in proper positions the desirable ion-cavity interactions can be achieved, and thus $W$ states of multi-ion (up to 10-ion) could be generated. Our numerical estimations showed that the existing fluctuations of the positions of the trapped ions and the velocity of the moving cavity do not 
significantly affect the fidelities of the generated $W$ states. We believe that the proposed model is scalable and might provide a new way to implement multi-qubit information processings, although moving the cavity is possibly a new challenge for the current technique.

This work is partly supported by the NSFC grant No. 10874142, 90921010, and the grant from the Major State Basic Research Development Program of China (973 Program) (No. 2010CB923104).

[1] Sackett C A, Kielpinski D, King B E, Langer C, Meyer V, Myatt C J, Rowe M, Turchette Q A, Itano W M, Wineland D J and Monroe C 2000 Nature 404256

[2] Zhao Z, Chen Y A, Zhang A N, Yang T, Briegel H J and Pan J W 2004 Nature 43054

[3] Leibfried D, Knill E, Seidelin S, Britton J, Blakestad R B, Chiaverini J, Hume D B, Itano W M, Jost J D, Langer C, Ozeri R, Reichle R and Wineland D J 2005 Nature 438639

[4] Häffner H, Hänsel W, Roos C F, Benhelm J, Chek-al-kar D, Chwalla M, Körber T, Rapol U D, Riebe M, Schmidt P O, Becher C, Ghühne O, Dür W and Blatt R, 2005 Nature 438643

[5] Buhrman H, Dam W V, Høyer P and Tapp A 1999 Phys. Rev. A 602737

[6] Dür W, Vidal G and Cirac J I 2000 Phys. Rev. A 62062314

[7] Leibfried D, Blatt R, Monroe C and Wineland D 2003 Rev. Mod. Phys. 75281

[8] Cirac J I and Zoller P 1995 Phys. Rev. Lett. 744091

[9] Mølmer K and Sørensen A 1999 Phys. Rev. Lett. 821835

[10] Wei L F, Liu S Y and Lei X L 2002 Phys. Rev. A 65062316

[11] Seidelin S, Chiaverini J, Reichle R, Bollinger J J, Leibfried D, Britton J, Wesenberg J H, Blakestad R B, Epstein R J, Hume D B, Itano W M, Jost J D, Langer C, Ozeri R, Shiga N and Wineland D J 2006 Phys. Rev. Lett. 96253003

[12] Raimond J M, Brune M and Haroche S 2001 Rev. Mod. Phys. 73565

[13] Schön C, Solano E, Verstraete F, Cirac J I and Wolf M M 2005 Phys. Rev. Lett. 95110503

[14] Keller M, Lange B, Hayasaka K, Lange W and Walther H 2004 Nature 4311075

[15] Guthöhrlein G R, Keller M, Hayasaka K, Lange W and Walther H 2001 Nature 41449

[16] Mundt A B, Kreuter A, Becher C, Leibfried D, Eschner J, Schmidt-Kaler F and Blatt R 2002 Phys. Rev. Lett. 89103001

[17] Keller M, Lange B, Hayasaka K, Lange W and Walther H 2003 J. Phys. B: At. Mol. Opt. Phys. 36613 
[18] Meyer G M, Löffler M, and Walther H 1997 Phys. Rev. A 56 R1099

[19] Meyer G M, Briegel H J, and Walther H 1997 Europhys. Lett. 37317 